\title{
Measurement of the top quark mass from leptonic observables in pp collisions
}

\author{
Cristina Mantilla Suarez ${ }^{* \dagger}$ \\ Florida Institute of Technology (US) \\ Escuela Politecnica Nacional (EC) \\ E-mail: cristina.ana.mantilla.suarez@cern.ch
}

\begin{abstract}
A novel technique for measuring the top quark mass using only leptonic observables is discussed. Top and anti-top quark decays with one electron and one muon and at least one jet in the final state are selected in proton-proton collision data collected by the CMS experiment at a center-of-mass energy of $8 \mathrm{TeV}$, corresponding to an integrated luminosity of $19.7 \mathrm{fb}^{-1}$. Several variables are studied and the transverse momentum distribution of the charged lepton pair originated from the top quark decay is chosen to extract the top quark mass. The measurement is calibrated using simulated events.
\end{abstract}

38th International Conference on High Energy Physics 3-10 August 2016

Chicago, USA

\footnotetext{
*Speaker.

${ }^{\dagger}$ On behalf of the CMS collaboration
} 


\section{Introduction}

The top quark mass $\left(\mathrm{m}_{t}\right)$ is a fundamental parameter of the standard model (SM) whose precise measurement can benefit the calculation of radiative corrections to several observables. The current $\mathrm{m}_{t}$ measurement methods exploit either the full kinematic reconstruction of top quark pair $(t \bar{t})$ events or alternative event topologies or observables that are sensitive to $\mathrm{m}_{t}$ variations. The latter can avoid intrinsic hadronization-related uncertainties and provide further understanding of the relation between the experimental results obtained for $\mathrm{m}_{t}$ and the mass parameters employed in theoretical calculations. In this report, we discuss a recent proposal to measure the top-quark mass from the kinematic properties of the dilepton system in $t \bar{t}$ events.

\section{Top quark mass measurement}

The observed distributions of the kinematic properties of the charged leptons depend on the dynamics of the top quark production and decay and can therefore be used to infer $\mathrm{m}_{t}$, as recently proposed in [1].

The sensitivity to theoretical assumptions and experimental effects that may affect this measurement was studied by carrying out the analysis for a set of $\mathrm{m}_{t}$-dependent observables reconstructed in the dilepton final state. The performance of the following kinematics was investigated separately: the transverse momentum, $p_{T}\left(l^{+}\right)$, and the energy, $E\left(l^{+}\right)$, of the positively-charged lepton; the transverse momentum $p_{T}\left(l^{+} l^{-}\right)$and the invariant mass $M\left(\ell^{+} \ell^{-}\right)$of the charged lepton pair; the scalar sum of the transverse momentum $p_{T}\left(l^{+}\right)+p_{T}\left(l^{-}\right)$and the energies $E\left(l^{+}\right)+E\left(l^{-}\right)$ of the two charged leptons. Among all the aforementioned observables, $p_{T}\left(l^{+} l^{-}\right)$is found to be the most robust against these effects and to be one of the most sensitive to $\mathrm{m}_{t}$. Hence, the $\mathrm{m}_{t}$ measurement reported is based solely on the results obtained with this variable.

The analysis focuses in $t \bar{t}$ events with one isolated electron and muon in the final state and uses the full dataset, collected by the CMS experiment [2] in proton-proton collisions at $\sqrt{s}=8 \mathrm{TeV}$, corresponding to an integrated luminosity of $19.7 \mathrm{fb}^{-1}$.

The $\mathrm{m}_{t}$ extraction is performed using the full shape of the distribution, and comparing the experimentally observed yields in individual bins with the prediction from fixed $\mathrm{m}_{t}$ hypothesis, ranging between 166.5 and $178.5 \mathrm{GeV}$, and using its first moments $\left(O^{(1)}, O^{(2)}\right)$ and comparing those extracted from data with the expected in simulated events. After subtracting the background contribution from data and applying the simulation-based calibration, the first moments are translated as $\mathrm{m}_{t}=171.8 \pm 1.2$ (stat) $\mathrm{GeV}\left(\mathrm{m}_{t}=171.6 \pm 1.2\right.$ (stat) $\left.\mathrm{GeV}\right)$. The shape analysis yields a value of $\mathrm{m}_{t}=171.7 \pm 1.1$ (stat) $\mathrm{GeV}$. The results are found to be in agreement with previous $\mathrm{m}_{t}$ measurements at CMS [3].

For each source of uncertainty, the size of the variation is estimated by using the alternative reconstructed prediction of the distribution to evaluate a difference in the moments of the distributions or in the estimator used to scan the $\mathrm{m}_{t}$ parameter (See Table 2). A detailed explanation of the sources of uncertainty can be found in [4].

The total experimental uncertainty is only limited by the lepton momentum scale calibration, while the dominant uncertainties stem from the modeling of top quark kinematics and the modeling of the hard process, which includes scale variations in the simulation, jet-to-parton matching, and 


\begin{tabular}{|c|c|c|c|}
\hline Source & $\Delta \mathrm{m}_{t}^{f i t}[\mathrm{GeV}]$ & $\Delta \mathrm{m}_{t}^{O^{(1)}}[\mathrm{GeV}]$ & $\Delta \mathrm{m}_{t}^{O^{(2)}}[\mathrm{GeV}]$ \\
\hline$\mu_{\mathrm{R}} / \mu_{\mathrm{F}}$ scales $\mathrm{t} \bar{t}$ & $\left\{\begin{array}{l}+1.61 \\
-2.29\end{array}\right.$ & $\left\{\begin{array}{l}+1.70 \\
-2.91\end{array}\right.$ & $\left\{\begin{array}{l}+1.79 \\
-2.71\end{array}\right.$ \\
\hline ME-PS matching scale & $\left\{\begin{array}{l}+0.91 \\
-1.06\end{array}\right.$ & $\left\{\begin{array}{l}+0.99 \\
-0.99\end{array}\right.$ & $\left\{\begin{array}{l}+1.08 \\
-0.79\end{array}\right.$ \\
\hline Parton density functions & \pm 0.35 & \pm 0.38 & \pm 0.37 \\
\hline Top quark $\mathrm{p}_{T}\left(p_{T}(\mathrm{t})\right)$ & +0.78 & +0.85 & +1.15 \\
\hline Underlying event & \pm 0.82 & \pm 0.56 & \pm 0.57 \\
\hline Color reconnection & \pm 0.48 & \pm 0.66 & \pm 0.51 \\
\hline Signal modeling & \pm 1.29 & \pm 0.71 & \pm 0.61 \\
\hline Jet energy scale & \pm 0.05 & \pm 0.05 & \pm 0.05 \\
\hline Jet energy resolution & $< \pm 0.01$ & $< \pm 0.01$ & $< \pm 0.01$ \\
\hline Lepton energy scale & \pm 0.37 & $\left\{\begin{array}{l}+0.24 \\
-0.48\end{array}\right.$ & $\left\{\begin{array}{l}+0.22 \\
-0.51\end{array}\right.$ \\
\hline Lepton selection efficiency & \pm 0.04 & \pm 0.08 & \pm 0.08 \\
\hline $\mathrm{b}$ tagging & \pm 0.02 & \pm 0.02 & \pm 0.02 \\
\hline Misidentification efficiency & \pm 0.03 & \pm 0.09 & \pm 0.09 \\
\hline Pileup & \pm 0.16 & \pm 0.24 & \pm 0.27 \\
\hline Background normalization & \pm 0.33 & \pm 0.45 & \pm 0.35 \\
\hline Total systematic & $+2.68-3.09$ & $+2.57-3.42$ & $+2.72-3.14$ \\
\hline
\end{tabular}

Table 1: Summary of the systematic uncertainties for the different $\mathrm{m}_{t}$ measurements performed in this analysis [4].

matrix-element generator. However, is expected that the use of next-to-leading (NLO) simulation in future analyses will improve these effects.

\section{Particle level correction}

In order to allow direct comparison with different generators and theoretical predictions, particlelevel distributions are reported for each of the aforementioned kinematic observables. An unfolding procedure is applied to correct the measured distributions for detector resolution effects which can lead to a migration of events across bin boundaries. The normalized unfolded distributions are reported in Fig. 1 and compared to predictions generated at different mass hypothesis $\left(\mathrm{m}_{t}=166.5,172.5,178.5 \mathrm{GeV}\right)$. Within the total uncertainties, good agreement is observed with respect to the nominal $\mathrm{m}_{t}=172.5 \mathrm{GeV}$ - based simulation.

\section{Conclusions}

A novel alternative technique to measure the mass of the top quark in $e \mu$ final states, with at least one $b$ jet, using the distribution of the transverse momentum of the charged lepton pair yields values of:

$$
\begin{gathered}
\mathrm{m}_{t}=171.8 \pm 1.2 \text { (stat.) }{ }_{-0.7}^{+0.6} \text { (exp.) }{ }_{-3.3}^{+2.4} \text { (th.) }{ }_{-0.0}^{+0.9}\left(p_{T} \text { (t) }\right) \mathrm{GeV} \text { (first moment) } \\
\mathrm{m}_{t}=171.6 \pm 1.2 \text { (stat.) }{ }_{-0.7}^{+0.5} \text { (exp.) }{ }_{-3.1}^{+2.4} \text { (th.) }{ }_{-0.0}^{+1.2}\left(p_{T}(\mathrm{t})\right) \mathrm{GeV} \text { (second moment) } \\
\mathrm{m}_{t}=171.7 \pm 1.1 \text { (stat.) } \pm 0.5 \text { (exp.) }{ }_{-3.1}^{+2.5} \text { (th. }{ }_{-0.0}^{+0.8}\left(p_{T}(\mathrm{t})\right) \mathrm{GeV} \text { (shape analysis) }
\end{gathered}
$$



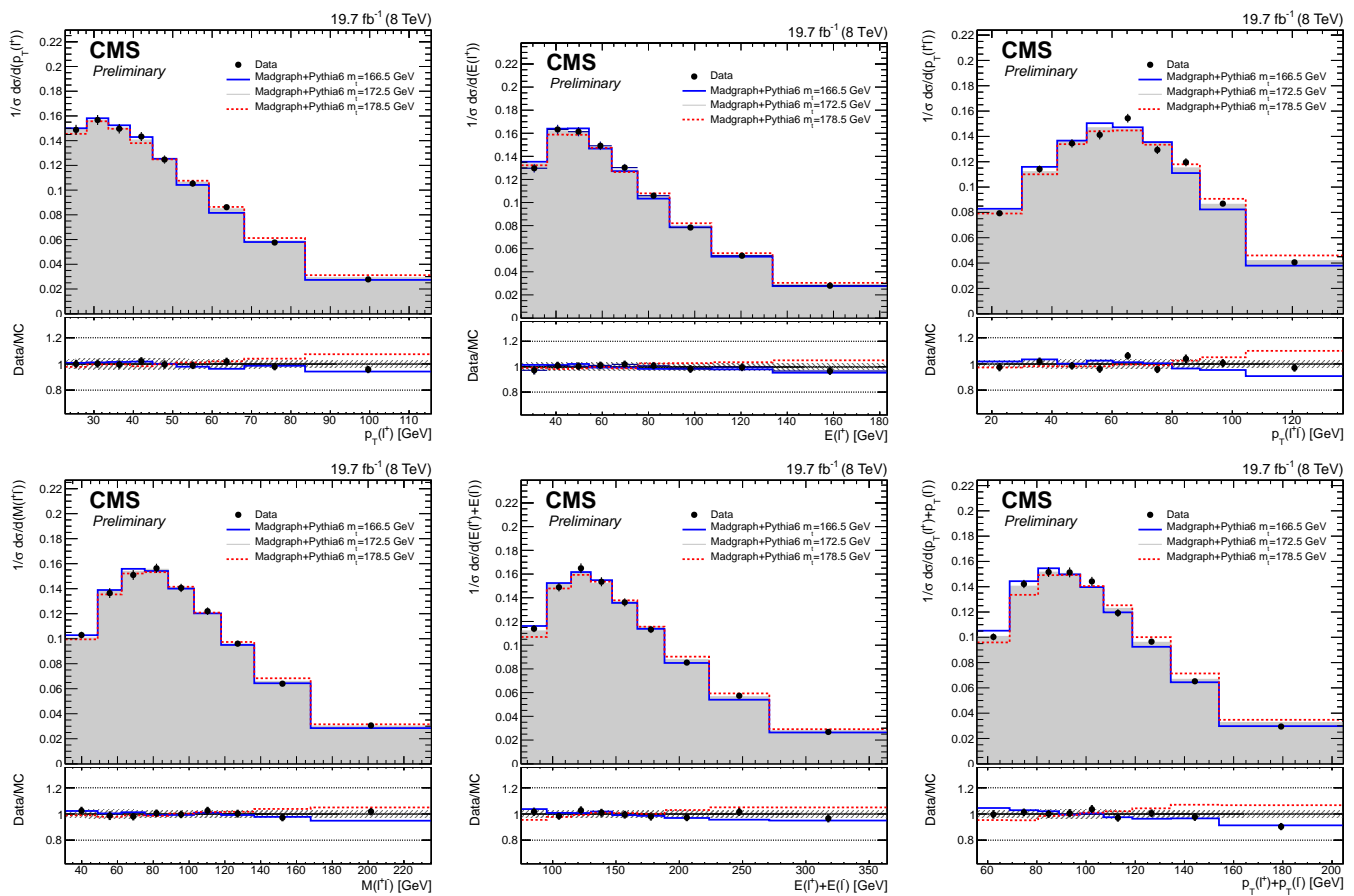

Figure 1: Normalized event yields at particle level for $t \bar{t}$ production, presented as a function of each of the kinematic distributions studied in this analysis [4]. The bottom panels show the ratio with respect to the nominal simulation generated at $\mathrm{m}_{t}=172.5 \mathrm{GeV}$ with the hatched band representing the envelope of the statistical and systematic uncertainties.

With improved MC event generators at NLO it is expected that the theoretical uncertainties, such as the scale choice in QCD calculations, will be significantly reduced allowing direct comparisons with the original theory proposal [1].

\section{References}

[1] S. Frixione and A. Mitov, "Determination of the top quark mass from leptonic observables", JHEP 09 (2014) 012, doi:10.1007/JHEP09(2014)012, arXiv:1407.2763.

[2] S. Chatrchyan et al. [CMS Collaboration], JINST 3 (2008) S08004. doi:10.1088/1748-0221/3/08/S08004

[3] E. Bouvier, "Selected top quark mass measurements at CMS", Proceedings, 38th International Conference on High Energy Physics: Chicago, USA, August 3-10, 2016.

[4] CMS Collaboration, "Determination of the top quark mass from leptonic observables using $e \mu+$ jets final states selected in proton-proton collisions at $\sqrt{s}=8 \mathrm{TeV}$ ", CMS-PAS-TOP-16-002. 